Abstract IDDF2018-ABS-0178 Table 1 Characteristics of each crss subtype of colorectal cancer

\begin{tabular}{|c|c|c|c|c|c|c|}
\hline & CRSS-A & CRSS-B & CRSS-C & CRSS-D & CRSS-E & CRSS-F \\
\hline Incidence & $23 \%$ & $23 \%$ & $12 \%$ & $8 \%$ & $25 \%$ & $10 \%$ \\
\hline Precursor lesions & $\begin{array}{l}\text { non-serrated } \\
\text { adenoma }\end{array}$ & non-serrated adenoma & serrated adenoma & non-serrated adenoma & serrated adenoma & serrated adenoma \\
\hline $\begin{array}{l}\text { Colon cell } \\
\text { derivation }\end{array}$ & crypt top, epithelial & crypt base, epithelial & crypt top, epithelial & crypt top, EMT & $\begin{array}{l}\text { crypt base, EMT, stem- } \\
\text { like }\end{array}$ & crypt base, EMT, stem-like \\
\hline $\begin{array}{l}\text { Transcriptomic } \\
\text { pathways }\end{array}$ & $\begin{array}{l}\text { glycolytic process } \\
\text { activation }\end{array}$ & $\begin{array}{l}\text { DNA replication } \\
\text { activation }\end{array}$ & IL-6 pathway activation & IL-2 pathway activation & $\begin{array}{l}\text { Immune pathways } \\
\text { activation }\end{array}$ & IGF1 pathway activation \\
\hline $\begin{array}{l}\text { Associated } \\
\text { mutations }\end{array}$ & KRAS & TP53 & hypermutation, BRAF & TP53 & KRAS, TP53 & hypermutation, KRAS \\
\hline $\begin{array}{l}\text { Stroma-immune } \\
\text { cells population }\end{array}$ & NK cells & $\begin{array}{l}\text { very few stroma and } \\
\text { immune cells enriches }\end{array}$ & $\begin{array}{l}\text { cytotoxic lymphocytes and } \\
\text { monocytic lineage }\end{array}$ & $\begin{array}{l}\text { many stroma and } \\
\text { immune cells enriched }\end{array}$ & $\begin{array}{l}\text { endothelial cells and } \\
\text { fibroblasts }\end{array}$ & $\begin{array}{l}\text { endothelial cells, fibroblasts } \\
\text { and monocytic lineage }\end{array}$ \\
\hline $\begin{array}{l}\text { Epigenomic } \\
\text { associations }\end{array}$ & $\begin{array}{l}\text { CIN(-), CIMP(-), } \\
\text { MSS }\end{array}$ & CIN(-), CIMP(-), MSS & $\mathrm{CIN}(+), \mathrm{CIMP}(+), \mathrm{MSI}$ & CIN(-), CIMP(-), MSS & CIN(-), CIMP(-), MSS & CIN(-), CIMP(-), MSS \\
\hline Location & distal & Distal & proximal & distal & distal & Mixed \\
\hline Prognosis & intermediate & Intermediate & good & intermediate & poor & Poor \\
\hline $\begin{array}{l}\text { Treatment } \\
\text { strategies }\end{array}$ & $\begin{array}{l}\text { EGFR inhibitors } \\
\text { (Cetuximab), 5-FU }\end{array}$ & $\begin{array}{l}\text { EGFR inhibitors } \\
\text { (Cetuximab), 5-FU }\end{array}$ & $\begin{array}{l}\text { tyrosine kinase inhibitor } \\
\text { (Genifitnib) }\end{array}$ & $\begin{array}{l}\text { FOLFIRI or FOLFOX } \\
\text { chemotherapy regimen }\end{array}$ & $\begin{array}{l}\text { FOLFIRI or FOLFOX } \\
\text { chemotherapy regimen }\end{array}$ & $\begin{array}{l}\text { FOLFIRI or FOLFOX } \\
\text { chemotherapy regimen }\end{array}$ \\
\hline
\end{tabular}

Background Gene expression profiling has been used to classify molecular subtypes in colorectal cancer (CRC). Given that tumour transcriptome signals not only derive from cancer cells but also from tumour microenvironment. Recent studies have shown that noncancerous components might affect the classification of CRC subtypes. We hypothesised that using stroma-specific gene signature would be more effective to identify CRC subtypes with clinical relevance.

Methods To this end, we analysed gene expression profiles from 1821 CRCs. We firstly constructed a signature where genes were both stroma-specifically expressed and associated with drug response. Further, we identified CRC stroma-specific subtypes (CRSS) using K-means clustering based on the signature and verified the classification in two datasets. We also used the nearest template prediction algorithm to predict drug response.

Results The CRSS subtypes were associated with distinct clinicopathological, molecular and phenotypic characteristics and specific enrichments of gene signatures and signalling pathways (table 1): (i) CRSS-A: non-serrated adenomas, colon crypt top derived, glycolytic, epithelial, KRAS-mutant, sensitive to Cetuximab, enriched with NK cells; (ii) CRSS-B: non-serrated adenomas, colon crypt base derived, DNA replication activity, epithelial, BRAF wild-type, TP53 mutant, chromosomal stability, distal CRC, sensitive to Cetuximab; (iii) CRSS-C: serrated adenomas, colon crypt top derived, interleukin-6 pathway activity, epithelial, microsatellite instability (MSI), BRAF mutant, hypermutation, chromosomal instability, CpG island methylator phenotype, proximal CRC, sensitive to Gefitinib, good prognosis, enriched with cytotoxic lymphocytes and monocytic lineage; (iv) CRSS-D: non-serrated adenomas, colon crypt top derived, interleukin-2 pathway activity, epithelialmesenchymal transition (EMT), chromosomal stability, sensitive to FOLFIRI and FOLFOX chemotherapy regimens; (v) CRSSE: serrated adenomas, colon crypt base derived, EMT, immune pathways activation, poor prognosis, sensitive to FOLFIRI and FOLFOX, enriched with endothelial cells and fibroblasts; (vi) CRSS-F: serrated adenomas, colon crypt base derived, EMT, IGF1 pathway activity, poor prognosis, sensitive to FOLFIRI and FOLFOX, enriched with endothelial cells, fibroblasts and monocytic lineage.
Conclusions We classified CRC into six molecular subtypes (CRSS). The identification of CRSS subtypes is critical, as it provides possibilities to identify robust prognostic models and provide more precise therapeutic options for each CRC subtype.

\section{IDDF2018-ABS-0194 ADIPOSE-DERIVED MESENCHYMAL STEM CELLS PROMOTE HEALING OF NSAID- RELATED GASTRIC ULCER THROUGH THE PARACRINE EFFECTS IN PIGS}

${ }^{1}$ Xianfeng Xia*, ${ }^{2}$ Kai Fung Chan, ${ }^{3}$ Gerald Tsz Yau Wong, ${ }^{4}$ Baldwin Po Man Yeung, ${ }^{5}$ Liu Liu, ${ }^{4}$ Enders Kwok Wai Ng, ${ }^{4}$ James Yun Wong Lau, ${ }^{4}$ Philip Wai Yan Chiu. ${ }^{1}$ Chow Yuk Ho Technology Center for Innovative Medicine, The Chinese University of Hong Kong, Hong Kong; ${ }^{2}$ Department of Biomedical Engineering, The Chinese University of Hong Kong, Hong Kong: ${ }^{3}$ Chow Tai Fook-Cheng Yu Tung Surgical Stem Cell Research Centre, The Chinese University of Hong Kong, Hong Kong; ${ }^{4}$ Department of Surgery, Institute of Digestive Disease, State Key Laboratory of Digestive Disease, The Chinese University of Hong Kong, Hong Kong; ${ }^{5}$ Department of General Surgery, The First Affiliated Hospital of University of Science and Technology of China, China

\subsection{6/gutjnl-2018-IDDFbestabstracts.4}

Background Long-term use of nonsteroidal anti-inflammatory drugs (NSAIDs) will induce severe gastrointestinal toxicity. Stem cell therapy is proposed to facilitate tissue repair and regeneration. This study aimed to investigate whether endoscopic submucosal injection of adipose-derived mesenchymal stem cells (ADMSCs) could accelerate healing of NSAID-related gastric ulcer.

Methods Porcine model of NSAID-related gastric ulcer was induced by indomethacin and endoscopic submucosal dissection (ESD)(Fig 1A). Indomethacin was given in all porcine models 10 days prior to ESD. We performed ESD at body and antrum of the stomach in each pig under general anaesthesia to create the ulcer model. After ESD, one of the ulcers was treated by endoscopic submucosal injection of ADMSCs, while the other ulcer was injected with saline as control. Further endoscopic injections were performed at respective ulcer on day 7 and 14. Indomethacin was continued after ESD until 
pigs were euthanized on day 21. Endoscopic surveillance was performed every week to measure the ulcer healing.

Results ADMSCs delivered by the endoscopic submucosal injection led to more efficient healing of NSAID-related gastric ulcer, with a significant wound closure observed by day 7 compared with the control (IDDF2018-ABS-0194 Figure 1. B, C). Ulcers in the ADMSCs group displayed enhanced re-epithelization and angiogenesis, and reduced inflammation on day 7 and 21 (IDDF2018-ABS-0194 Figure 1. D-G). Moreover, few engrafted ADMSCs showed myofibroblast and epithelial cell phenotype in vivo, suggesting the stem cell-assisted ulcer healing may not rely on cell differentiation (IDDF2018-ABS0194 Figure 1. H, I). A further experiment with submucosal injection of MSC-derived secretome revealed that the therapeutic efficacy of NSAID-related gastric ulcer was comparable to stem cell transplantation (IDDF2018-ABS-0194 Figure 2. A, B). Profiling analysis showed significant up-regulation of genes associated with inflammation, granulation and extracellular matrix remodelling (IDDF2018-ABS-0194 Figure 2. C, D). In addition, the ErK1/2-MAPK pathway was activated by injection of ADMSCs or MSC-derived secretome (IDDF2018-ABS0194 Figure 2. E, F).

Conclusions Our results showed that endoscopic submucosal injection of ADMSCs served as a novel approach to promote healing of NSAID-related gastric ulcer, while the paracrine activity of stem cell played a more important role in this process (IDDF2018-ABS-0194 Figure 2. G).

\section{Basic Hepatology}

\section{IDDF2018-ABS-0037 THERAPEUTIC BENEFIT OF ADIPOSE- DERIVED STROMAL VASCULAR FRACTION CELL TRANSPLANTATION IN RATS OF ACUTE-ON-CHRONIC LIVER FAILURE}

${ }^{1}$ Cheng-Maw Ho* ${ }^{1}$ Ya-Hui Chen, ${ }^{2}$ Chin-Sung Chien, ${ }^{1}$ Shu-Li Ho, ${ }^{3}$ Chih-Chiang Hsiao, ${ }^{3}$ Jung-Cheu Chen, ${ }^{1}$ Hui-Ling Chen, ${ }^{1}$ Rey-Heng Hu, ${ }^{3}$ Daniel Tzu-bi Shih, ${ }^{1}$ Po-Huang Lee. ${ }^{1}$ National Taiwan University Hospital, Taiwan; ${ }^{2}$ National Taiwan University, Taiwan; ${ }^{3}$ Taipei Medical University, Taiwan

\subsection{6/gutjnl-2018-IDDFbestabstracts.5}

Background Adipose-derived stromal vascular fraction (SVF) cells, a rich source of primary stem/stromal cells, are promising for administering cell therapy for patients with acute-on-chronic liver failure (ACLF). We evaluated the therapeutic effects of CD34+/CD34- SVF cells in hepatocyte cotransplantation in a rat model of ACLF.

Methods ACLF was induced in Sprague-Dawley rats by temporary bile duct ligation and d-galactosamine administration. Donor hepatocytes and SVF cells (labelled with the PKH26 fluorescent dye) were freshly isolated from $\mathrm{Tg}$ (UBC-emGFP) rats and human adipose tissues, respectively. Sorted CD34 tand CD34- SVF cells were separated using a magnetic bead system. Rat hepatocytes and SVF cells (unsorted and sorted) were intraportally transplanted into ACLF rats. The surviving animals were sacrificed at 1 and 2 weeks after transplantation. Results ACLF was evidenced by the development of acute coagulopathy and hepatocyte necrosis in fibrotic livers. Compared with the control group, the unsorted group showed less biliary ductular proliferation and fibrosis at 1 week after transplantation. Prominent biliary ductular proliferation and significantly increased fibrosis were observed in the CD34- group than in the CD34 +group at 1 week. At 2 weeks, the serum level of alkaline phosphatase was significantly lower in the CD34 +group than in the CD34- group. The transplanted SVF cells were found in the periportal regions at 1 week, whereas donor hepatocytes were rarely detected.

Conclusions Compared with CD34- SVF cells, cotransplantation of CD34 +SVF cells resulted in the early amelioration of liver fibrosis and biliary ductular proliferation in ACLF rats.

\section{IDDF2018-ABS-0097 GENOMIC HETEROGENEITY AND CLONAL EVOLUTION OF MULTIFOCAL HEPATOCELLULAR CARCINOMA}

${ }^{1}$ Lixia Xu*, ${ }^{2}$ Minghui He, ${ }^{2}$ Zihao Dai, ${ }^{1}$ Sui Peng, ${ }^{2}$ Ming Kuang. ${ }^{1}$ Department of Gastroenterology, The First Affiliated Hospital of Sun Yat-sen University, Guangzhou, China; ${ }^{2}$ Department of Liver Surgery, The First Affiliated Hospital of Sun Yat-sen University, China

\subsection{6/gutjnl-2018-IDDFbestabstracts.6}

Background Hepatocellular carcinoma (HCC) often presents with multiple tumours within the liver. Although recent genomic studies evaluating intratumor heterogeneity, the identification of intrapatient heterogeneity (IPH) and its impact on targeted therapy for multifocal HCC remain unclear. We aimed to characterise genomic architecture, infer the clonal evolution of multifocal HCC, and explore whether genomic profiling could help select suitable patients for targeted therapy.

Methods 43 tumour samples from 11 multifocal HCC patients were employed for whole-genome sequencing, whole-exome sequencing and RNA sequencing. Somatic mutations, copy number alterations (CNAs), structural variations (SVs), hepatitis B virus integrations, as well as clonal evolution and druggable targets of each tumour were analysed.

Results We identified substantial IPH in mutational profiles, CNAs, SVs and clonal evolution among tumours separated by anatomical locations within the same patient. 41 out of 43 tumour samples in our study belong to intrahepatic metastasis. Phylogenetic analysis found that only $31 \%$ of the driver alterations were truncal events, indicating evident IPH and branched tumour evolution of multifocal HCCs. Moreover, only $23 \%$ of druggable alterations were mapped to trunks. Of note, sorafenib sensitive targets were identified in the trunk of only one out of five patients.

Conclusions Our study provided a detailed view of IPH and clonal evolution of multifocal HCC. Most tumours in our cohort belong to intrahepatic metastasis and follow the scenario of branched tumour evolution. Majority of druggable targets are non-truncal alterations, emphasising the importance of multiple-tumor-sampling of all foci from multifocal HCC patients for personalised targeted therapies.

\section{IDDF2018-ABS-0153 SUPER-ENHANCER-ASSOCIATED MASTER TRANSCRIPTIONAL CIRCUITRY IN NAFLD- HCC DEVELOPMENT}

${ }^{1}$ Otto Ka Wing Cheung ${ }^{*},{ }^{2}$ Feng Wu, ${ }^{3}$ Sau-Dan Lee, ${ }^{4}$ Patrick Tan, ${ }^{3}$ Kevin Yuk-Lap Yip, ${ }^{2}$ Ka Fai To, 'Alfred Sze-Lok Cheng. 'School of Biomedical Sciences, The Chinese University of Hong Kong, Hong Kong; ' 2 Department of Anatomical and Cellular Pathology, The Chinese University of Hong Kong, Hong Kong; ${ }^{3}$ Department of Computer Science and Engineering, The Chinese University of Hong Kong, Hong Kong; ${ }^{4}$ Duke-NUS Graduate Medical School, Singapore

10.1136/gutjnl-2018-IDDFbestabstracts.7 